\title{
PENDULUM COINCIDENCES DETERMINED BY SHADOW REËNFORCEMENTS, SHADOW MICROMETRY, ETC.
}

By C. Barus.

I. Let two similar pendulums be mounted, one normally behind the other relatively to their nearly parallel planes of vibration. Provide each (light cork attachment and incision) with a small square of wire gauze (one inch square) of about a millimeter mesh, the planes of the gauze being nearly normally behind each other, about six inches apart, and parallel to the plane of vibration. Let the threads of the gauze be respectively horizontal and vertical (or in general nearly parallel in the two cases) and let the pendulums be swung over about the same arc.

2. When the pendulums are at rest, the observer looking from a distance through one gauze at the other with a bright background behind it (white paper, spot of sunlight, ground glass and lamp), sees the usual shadow reënforcements, in form of more or less equidistant horizontal and vertical dark bands. When the pendulums move the vertical bands are usually wiped out except at the elongations, because the corresponding wires of the gauze sweep past each other too rapidly to be recorded by the eye. The horizontal bands, however, remain in the field, for there is no translation in the direction of the axis of a pendulum; but there is rotation. Hence, as the pendulums sweep over any angle $\alpha$ with respect to each other, the originally horizontal shadow bands sweep over a large multiple of $\alpha$. Thus the shadow bands are seen to topple from an angle of more than 45 degrees above the horizontal, to more than 45 degrees below it, except at the moment of coincidence, when they are for an instant stationary and nearly horizontal. At this instant the hands of a stop watch may be released and arrested again at the next coincidence.

If $\alpha$ be the angle between the nearly horizontal wires of the gauze 
and $\gamma$ the angle of the shadow bands with the direction of either of the wires; if $a$ is the distance apart of the wires and $b$ the projection of the $a$ of one on the plane of the other by the eye of the ob. server, so that $a$ and $b$ are the virtual distances apart of the effective wires, we may write $\tan \gamma=\alpha \tan \alpha /(\alpha-b \sec \alpha)$, and thus $\gamma$ is liable to be greater than 90 degrees. If $\alpha$ is small tan $\gamma=$ $\alpha \alpha /\left[(a-b)-\alpha \alpha^{2} / 2\right]$ and for very small $\alpha, \tan \gamma=\alpha \alpha /(a-b)$. Since $a$ and $b$ are nearly equal the rapid increase of $\tan \gamma$ as $\alpha$ passes through zero is manifest.

3. The phenomenon is very beautiful. With a larger square of wire gauze ( 6 inches square and $\frac{1}{8}$-inch mesh) it may be easily shown to a class on a light background. When the two pendulums are all but stationary, the horizontal bands are also nearly so, while the vertical bands are now permanently in the field magnifying the motion and becoming fixed at the coincidences. Pendulums may be thus compared which are scarcely seen to move, though for this purpose the method of the next paragraph is still better. Finally the phenomenon may be projected in various ways, but in no case are the results so satisfactory as the above. If two pendulums vibrate at right angles to each other, the elongations of the vertical pendulum are useful in the same way, horizontal bands recurring and marking the coincidences of the upper elongation of the vertical pendulum and the mean position of the horizontal pendulum.

4. The most fascinating reinforcements are obtainable from the superposition of two identical sets of concentric circles, which may be drawn or photographed on glass plates. If the circles are equidistant the hyperbolæ of Young's interferences are strikingly brought out for all distances apart of the centers. If these shadow bands are to be straight lines, equidistant and at right angles to the lines joining the centers of the two plates, the $n$th and $(n+\mathrm{I})$ th radii of the circles of each of the identical plates in the $m$ th black band must be compatible with $r_{m+n+1}{ }^{2}-{r_{n+1}}^{2}=r_{m+n}{ }^{2}-r_{n}^{2}$ where $r_{n}$ and $r_{m+n}, r_{n+1}$ and $r_{m+n+1}$ are the radii from the centers, respectively, to the two consecutive intersections on the same dark band. Hence the radii may be put $r_{n}=\sqrt{n}$; or the linear bands are obtained by superposing two zone plates. A disk of about one half centimeter in diameter or larger is satisfactory, 
To use this arrangement as a micrometer, suppose the distances to be measured by the relative motion of the two superposed plates are in a horizontal direction, and let $x$ be one of these small distances. Let the plates be placed in a vertical plane through $x$, and so that the center, $c$, of one set of circles may be $a$ centimeters above the center, $c^{\prime}$, of the other set of circles. Then if $\gamma$ is the angle of the dark bands (normal to $c c^{\prime}$ ) with the horizontal $x$, $\tan \gamma=x / a$. Thus if $a$ is one tenth millimeter, a displacement of $x$ will rotate the originally horizontal bands ( $c c^{\prime}$ vertical), rising or falling according to the relative motion, roughly about as follows :

$$
\begin{array}{rrrrrr}
x & =.002 & .005 & .0 \mathrm{I} & .03 & \text {. I millimeter } \\
- \pm r & =\mathrm{I}^{\circ} & 3^{\circ} & 6^{\circ} & \mathrm{I} 7^{\circ} & 45^{\circ}
\end{array}
$$

The distance apart of the bands is uniformly $\mathrm{I} / 2 \sqrt{x^{2}+a^{2}}$ or $\mathrm{I} / 2\left(x^{2}+a^{2}\right)$ of the distance apart of centers; and they thus become wider as $x$ and $a$ decrease, vanishing at infinity in case of coincidence. Finally if a medium could be constructed in which the index of refraction increases about as the normal distance from the lines joining the centers, the present phenomenon would approximately replace Young's interferences.

BROWN UNIVERSITY,

Providence, R. I 